\title{
REGULARITY OF ENTROPY SOLUTIONS OF QUASILINEAR ELLIPTIC PROBLEMS RELATED WITH HARDY-SOBOLEV INEQUALITIES
}

\author{
BOUMEDIENE ABDELLAOUI, EDUARDO COLORADO AND MANEL SANCHÓN
}

ABSTRACT: This article is concerned with the regularity of the entropy solution of

$$
\left\{\begin{aligned}
-\operatorname{div}\left(|x|^{-\gamma p}|\nabla u|^{p-2} \nabla u\right) & =f(x) & & \text { in } \Omega, \\
u & =0 & & \text { on } \partial \Omega,
\end{aligned}\right.
$$

where $\Omega$ is a smooth bounded domain $\Omega$ of $\mathbb{R}^{N}$ such that $0 \in \Omega, 1<p<N$, and $\gamma<(N-p) / p$. Assuming $f \in L^{q}\left(\Omega,|x|^{\alpha(q-1)} d x\right)$ for some $q \geq 1$ and $N \gamma p /(N-p) \leq$ $\alpha \leq(\gamma+1) p$, we obtain estimates for the entropy solution $u$ and its weak gradient in Lebesgue spaces with weights. Moreover, we introduce some explicit examples showing the optimality of our results and a relation between our problem and a Hardy-Sobolev type inequality.

AMS SubJECT ClassificATION (2000): 35D10, 35J25; 35J70; 46E35 .

\section{Introduction.}

Let $\Omega$ be a smooth bounded domain of $\mathbb{R}^{N}$ such that $0 \in \Omega$. Let $1<p<N$ and $-\infty<\gamma<(N-p) / p$. We consider the quasilinear elliptic problem

$$
\left\{\begin{array}{rlrl}
-\operatorname{div}\left(|x|^{-\gamma p}|\nabla u|^{p-2} \nabla u\right) & =f(x) & \text { in } \Omega, \\
u & = & 0 & \text { on } \partial \Omega .
\end{array}\right.
$$

Throughout the paper we assume that $f$ belongs to the Banach space $\left(\mathcal{E}_{\alpha}^{q}(\Omega),\|\cdot\|_{\alpha, q}\right)$, for some $q$ and $\alpha$ satisfying

$$
q \geq 1 \quad \text { and } \quad \frac{N \gamma p}{N-p} \leq \alpha \leq(\gamma+1) p,
$$

where

$$
\mathcal{E}_{\alpha}^{q}(\Omega):=\left\{f:|x|^{\alpha} f \in L^{q}\left(\Omega,|x|^{-\alpha} d x\right)\right\} \equiv L^{q}\left(\Omega,|x|^{\alpha(q-1)} d x\right)
$$

and

$$
\|f\|_{(\alpha, q)}:=\|f\|_{L^{q}\left(\Omega,|x|^{\alpha(q-1)} d x\right)} .
$$

Received June 26, 2006.

The first author is supported by the MEC Spanish grant MTM2004-02223. The second author is partially supported by the MEC Spanish grants MTM2004-02223 and BFM2003-03772. The third author is supported by CMUC/FCT (Coimbra, Portugal), and the MCYT and MEC Spanish grants BMF2002-04613C03 and MTM2005-07660-C02-01. 
Problem (1) and assumption (2) are related with the following Hardy-Sobolev inequality which is an immediate consequence of some well known CaffarelliKohn-Nirenberg inequalities (see [8]).

Lemma 1.1. Let $r \geq 1$ and $\alpha, \gamma \in \mathbb{R}$ such that $\alpha<N$ and $\gamma<(N-r) / r$. Let

$$
r_{\alpha, \gamma}^{*}:=\frac{(N-\alpha) r}{N-(\gamma+1) r} .
$$

There exists a positive constant $D$ depending only on the parameters and independent of $\Omega$ such that

$$
\left(\int_{\Omega}|\phi|^{r_{\alpha, \gamma}^{*}}|x|^{-\alpha} d x\right)^{1 / r_{\alpha, \gamma}^{*}} \leq D\left(\int_{\Omega}|\nabla \phi|^{r}|x|^{-\gamma r} d x\right)^{\frac{1}{r}}
$$

for all $\phi \in \mathcal{C}_{0}^{\infty}(\Omega)$, if and only if

$$
\alpha-r \leq \gamma r \leq \frac{(N-r) \alpha}{N}
$$

We note that for $\alpha, \gamma=0$ the previous inequality reduces to the classical Sobolev inequality. For $\alpha=(\gamma+1) r$ one obtains the Hardy-Sobolev inequality (if in addition $\gamma=0$ and $r=2$ one has the classical Hardy inequality). In the last section of this paper we give some examples related to these inequalities and problem (1).

The energy setting for problem (1) is the weighted Sobolev space $\mathcal{D}_{0, \gamma}^{1, p}(\Omega)$, which is defined as the completion of $\mathcal{C}_{0}^{\infty}(\Omega)$, with respect to the norm

$$
\|\phi\|_{\gamma, p}:=\left(\int_{\Omega}|\nabla \phi|^{p}|x|^{-\gamma p} d x\right)^{1 / p} .
$$

However, this variational setting requires that the right-hand side $f$ will be in the dual space $\mathcal{D}_{-\gamma}^{-1, p^{\prime}}(\Omega)$ of $\mathcal{D}_{0, \gamma}^{1, p}(\Omega)$. Under this requirement it is easy to obtain the existence and uniqueness of a weak energy solution $u \in \mathcal{D}_{0, \gamma}^{1, p}(\Omega)$ using the classical results developed in the sixties by Browder and Minty (see [15]). By a weak energy solution of (1) we mean a function $u \in \mathcal{D}_{0, \gamma}^{1, p}(\Omega)$ such that

$$
\int_{\Omega}|\nabla u|^{p-2} \nabla u \cdot \nabla \phi|x|^{-\gamma p} d x=\int_{\Omega} f \phi d x \quad \text { for all } \phi \in \mathcal{C}_{0}^{\infty}(\Omega) .
$$


As a consequence of Lemma 1.1 we have that $\mathcal{E}_{\alpha}^{q}(\Omega) \subset \mathcal{D}_{-\gamma}^{-1, p^{\prime}}(\Omega)$ for all

$$
q \geq \tilde{q}:=\frac{(N-\alpha) p}{(p-1) N+(\gamma+1-\alpha) p} \quad \text { and } \quad \frac{N \gamma p}{N-p} \leq \alpha \leq(\gamma+1) p
$$

(see Lemma 2.3 below). Therefore, if $f \in \mathcal{E}_{\alpha}^{q}(\Omega)$, with $\alpha$ and $q$ satisfying (4), then there exists a unique weak energy solution $u \in \mathcal{D}_{0, \gamma}^{1, p}(\Omega)$ of (1).

Remark 1.2. The regularity theory of finite energy solutions of elliptic equations in divergence form, has been investigated by brilliant mathematicians of XX century. The first works by De Giorgi in 1957, [10], and Nash in 1958, [18]. Later, appeared the papers by Moser [16], [17] with different proofs. Some more relevant results were given by Stampacchia, Ladyzhenskaya-Ural'tseva, Serrin, etc.

Closer to the regularity of the problems we study here, are the $\mathcal{C}^{\kappa}$ regularity results studied in [12] for the linear case: $p=2,-\infty<\gamma<(N-2) / 2$, and in [9] for the nonlinear case: $1<p<N,-\infty<\gamma<(N-p) / p$, where, among other results, is proved that if $f$ satisfy

$$
\int_{\Omega}|f|^{r}|x|^{\frac{N \gamma p}{N-p}(r-1)} d x<\infty
$$

for some $r>N / p$, then $u \in L^{\infty}(\Omega)$ and moreover, $u \in \mathcal{C}^{\kappa}(\bar{\Omega})$ for some $0<\kappa<$ 1. Observe that the previous $L^{\infty}$ result is covered in Theorem 1.3 (i) below.

For $f \notin \mathcal{D}_{-\gamma}^{-1, p^{\prime}}(\Omega)$ problem (1) does not admit any weak energy solution. However there exists a new framework for which problem (1) is solvable. More precisely, defining the truncation function $T_{t}$ by

$$
T_{t}(s):=\max \{-t, \min \{t, s\}\}, \quad s \in \mathbb{R},
$$

we say that a measurable function $u$ is an entropy solution of (1) if $T_{t}(u) \in$ $\mathcal{D}_{0, \gamma}^{1, p}(\Omega)$, for every $t>0$, and

$$
\int_{\Omega}|\nabla u|^{p-2} \nabla u \cdot \nabla T_{t}(u-v)|x|^{-\gamma p} d x=\int_{\Omega} f T_{t}(u-v) d x,
$$

for every $t>0$, and for every $v \in \mathcal{D}_{0, \gamma}^{1, p}(\Omega) \cap L^{\infty}(\Omega)$. We note that a measurable function $u$ such that $T_{t}(u) \in \mathcal{D}_{0, \gamma}^{1, p}(\Omega)$, for every $t>0$, does not necessarily belong to $W_{0}^{1,1}(\Omega)$. However, it is possible to define its weak gradient (see Lemma 2.4), still denoted by $\nabla u$.

Bénilan et al. [3] introduced this notion of solution to problem (1) for $\gamma=0$ and $f \in L^{1}(\Omega)$. They proved the existence and uniqueness of an entropy solution 
to problem (1). Recently, Peral and one of the authors [1] extended these results to every $\gamma<(N-p) / p$. Therefore, we may assume the existence of a unique entropy solution to (1), since $\mathcal{E}_{\alpha}^{q}(\Omega) \subset L^{1}(\Omega)$ for all $\alpha$ and $q$ satisfying (2). Our main purpose here is to study the regularity of such a solution.

The first result that we prove concerns to the regularity of the entropy solution in some appropriate Lebesgue spaces with weights.

Theorem 1.3. Assume $f \in \mathcal{E}_{\alpha}^{q}(\Omega)$ for some $\alpha$ and $q$ satisfying (2). Let

$$
|\Omega|_{\alpha}:=\int_{\Omega}|x|^{-\alpha} d x \quad \text { and } \quad r_{1}:=\frac{(p-1)(N-\alpha) q}{N-\alpha-(\gamma p+p-\alpha) q} .
$$

There exists a positive constant $C$, depending on $N, \alpha, \gamma$, and $p$ such that if $u$ is the entropy solution of (1) then the following assertions hold:

(i) If $\alpha<(\gamma+1) p$ and $q>(N-\alpha) /(\gamma p+p-\alpha)$, then $u \in L^{\infty}(\Omega)$. Moreover,

$$
\|u\|_{\infty} \leq C\|f\|_{(\alpha, q)}^{\frac{1}{p-1}}|\Omega|_{\alpha}^{-1 / r_{1}}\left(-r_{1}\right) .
$$

(ii) If $\alpha<(\gamma+1) p$ and $q=(N-\alpha) /(\gamma p+p-\alpha)$, then $u \in L^{r}\left(\Omega,|x|^{-\alpha} d x\right)$, for all $1 \leq r<+\infty$. Moreover,

$$
\left(\int_{\Omega}|u|^{r}|x|^{-\alpha} d x\right)^{\frac{1}{r}} \leq C|\Omega|_{\alpha}^{\frac{1}{r}}\|f\|_{(\alpha, q)}^{\frac{1}{p-1}}, \quad \text { for all } 1 \leq r<+\infty .
$$

(iii) If either, $\alpha=(\gamma+1) p$, or $\alpha<(\gamma+1) p$ and $1 \leq q<(N-\alpha) /(\gamma p+p-\alpha)$, then $|u|^{r} \in L^{1}\left(\Omega,|x|^{-\alpha} d x\right)$, for all $0<r<r_{1}$. Moreover,

$$
\left(\int_{\Omega}|u|^{r}|x|^{-\alpha} d x\right)^{\frac{1}{r}} \leq C|\Omega|_{\alpha}^{\frac{1}{r}-\frac{1}{r_{1}}}\|f\|_{(\alpha, q)}^{\frac{1}{p-1}}, \quad \text { for all } 0<r<r_{1},
$$

where in this case the constant $C$ depending also on $q$.

In order to prove this theorem we take an adequate test function in the entropy condition (5). Then, using the Hardy-Sobolev inequality (3) with $r=1$, we obtain a differential inequality for the distribution function of $u$ with weight $|x|^{-\alpha}$. Finally, solving this inequality in the different cases of the theorem, we prove the result. This kind of argument were used by Talenti [19] to obtain some a priori estimates for weak energy solutions when $\alpha=\gamma=0$. Recently, Grenon [13] and Alvino et al. [2] proved Theorem 1.3 for $\alpha=\gamma=0$ in a similar way.

Our next result concerns to the regularity of the weak gradient of the entropy solution $u$ of (1) when $1 \leq q<\tilde{q}$. As we said above, if $q \geq \tilde{q}$ then there exists a weak energy solution $u \in \mathcal{D}_{0, \gamma}^{1, p}(\Omega)$. In this last case, higher integrability results 
are known for the gradient of $u$ when $\alpha=\gamma=0$ (see [11] and [14]). However, to our knowledge, the techniques used here does not apply in this case.

Theorem 1.4. Let $\tilde{q}$ be defined in (4) and

$$
\bar{q}:=\frac{N-\alpha}{(p-1)(N-\alpha)+\gamma+1-\alpha / p} .
$$

Assume $f \in \mathcal{E}_{\alpha}^{q}(\Omega)$ for some $\alpha$ and $q$ satisfying (2). If $u$ is the entropy solution of (1) then it holds that:

(i) If $\max \{1, \bar{q}\}<q<\tilde{q}$ then $u \in \mathcal{D}_{0, \beta}^{1, r_{2}}(\Omega)$, where

$$
r_{2}:=\frac{(p-1)(N-\alpha) q}{N-\alpha-(\gamma+1-\alpha / p) q} \quad \text { and } \quad \beta:=\gamma+\frac{\alpha}{r_{2}}-\frac{\alpha}{p} .
$$

In particular, $u \in L^{r_{1}}\left(\Omega,|x|^{-\alpha} d x\right)$, where $r_{1}$ is defined in (6).

(ii) If $1 \leq q \leq \max \{1, \bar{q}\}$ then there exists a constant independent of $u$ such that

$$
\int_{\Omega}|\nabla u|^{r}|x|^{-\left(\gamma+\frac{\alpha}{r}-\frac{\alpha}{p}\right) r} d x \leq C \quad \text { for all } 0<r<r_{2} .
$$

Part (i) extends the regularity results by Boccardo and Gallouët $[4,5]$ for $\alpha=$ $\gamma=0$. We prove the general case in a similar way, but using the Hardy-Sobolev inequality (3) instead of the classical Sobolev inequality. Alvino et al. [2] proved part (ii) (and also part (i)) for $\alpha=\gamma=0$. The proof in the general case uses the estimates obtained, in order to prove Theorem 1.3, for the distribution function of $u$ with weight $|x|^{-\alpha}$.

Remark 1.5. First, we note that the last assertion in Theorem 1.4(i) is an immediate consequence of Lemma 1.1 with $r=r_{2}$ and replacing $\gamma$ by $\beta$, since

$$
r_{1}=\frac{(N-\alpha) r_{2}}{N-(\beta+1) r_{2}}
$$

and all the assumptions in the lemma hold by (2). On the other hand, if $q=\tilde{q}$ then $r_{2}=p$ and $\beta=\gamma$, and for $q=\bar{q}$ one has $r_{2}=1$ and $\beta=\gamma-\alpha / p^{\prime}$.

Now, we do some comments for the critical case $\alpha=\gamma p+p$. By Theorem 1.3, if $f \in \mathcal{E}_{\alpha}^{q}(\Omega)$, for some $q \geq 1$, then $|u|^{r} \in L^{1}\left(\Omega,|x|^{-\alpha} d x\right)$ for all $r<r_{1}=(p-1) q$. In particular, if $q=+\infty$ we obtain $|u|^{r} \in L^{1}\left(\Omega,|x|^{-\alpha} d x\right)$ for all $1 \leq q<+\infty$. However, in this special case it remains open to prove that $u$ is a bounded solution. 
Finally, under the assumptions of Theorem 1.4, one has that $r_{1}=r_{2}$ whenever $\alpha=\gamma p+p$, and hence, we obtain $|x|^{r}|\nabla u|^{r} \in L^{1}\left(\Omega,|x|^{-\alpha} d x\right)$ for all $r<(p-1) q$.

The paper is organized as follows. In section 2 we give the necessary tools to prove our results. Section 3 concerns to the proof of Theorem 1.4. In section 4 we prove Theorem 1.4. Finally, section 5 deals with some explicit examples which show the optimality of our results and the relation between problem (1) and the Hardy-Sobolev inequality (3).

\section{Preliminaries}

We start this section recalling the Caffarelli-Kohn-Nirenberg inequalities (see $[8])$.

Lemma 2.1. Let $p, q, r, \beta, \sigma, \gamma$, and a be real constants such that $p, q \geq 1, r>0$, $0 \leq a \leq 1$, and

$$
\frac{1}{r}+\frac{\alpha}{N}, \frac{1}{p}+\frac{\gamma}{N}, \frac{1}{q}+\frac{\beta}{N}>0
$$

where $\alpha=a \sigma+(1-a) \beta$. There exists a positive constant $C$, depending only on the parameters, such that the following inequality holds for all $\phi \in \mathcal{C}_{0}^{\infty}\left(\mathbb{R}^{N}\right)$

$$
\left\||x|^{\alpha} \phi\right\|_{L^{r}\left(\mathbb{R}^{N}\right)} \leq C\left\||x|^{\gamma}|\nabla \phi|\right\|_{L^{p}\left(\mathbb{R}^{N}\right)}^{a}\left\||x|^{\beta} \phi\right\|_{L^{q}\left(\mathbb{R}^{N}\right)}^{1-a},
$$

if and only if the following relations hold:

$$
\begin{gathered}
\frac{1}{r}+\frac{\alpha}{N}=a\left(\frac{1}{p}+\frac{\gamma-1}{N}\right)+(1-a)\left(\frac{1}{q}+\frac{\beta}{N}\right), \\
0 \leq \gamma-\sigma \quad \text { if } \quad a>0,
\end{gathered}
$$

and

$$
\gamma-\sigma \leq 1 \quad \text { if } \quad a>0 \quad \text { and } \quad \frac{1}{r}+\frac{\alpha}{N}=\frac{1}{p}+\frac{\gamma-1}{N} .
$$

As we said in the introduction, Lemma 1.1 is an immediate consequence of Lemma 2.1 and hence we omit its proof. We write Lemma 1.1 for $r=1$ since it will be a key point in the proof of most of our results.

Lemma 2.2. Let $\alpha, \gamma \in \mathbb{R}$ such that $\alpha<N$ and $\gamma<N-1$. There exists a positive constant $D$, depending only on the parameters, such that

$$
\left(\int_{\Omega}|\phi|^{\frac{N-\alpha}{N-(\gamma+1)}}|x|^{-\alpha} d x\right)^{\frac{N-(\gamma+1)}{N-\alpha}} \leq D \int_{\Omega}|\nabla \phi||x|^{-\gamma} d x
$$


for all $\phi \in \mathcal{C}_{0}^{\infty}(\Omega)$, if and only if

$$
\alpha-1 \leq \gamma \leq \frac{(N-1) \alpha}{N}
$$

Another consequence of Lemma 1.1 is the following inclusion.

Lemma 2.3. $\mathcal{E}_{\alpha}^{q}(\Omega) \subset \mathcal{D}_{-\gamma}^{-1, p^{\prime}}(\Omega)$ for all

$$
q \geq \tilde{q}=\frac{(N-\alpha) p}{(p-1) N+(\gamma+1-\alpha) p} \quad \text { and } \quad \frac{N \gamma p}{N-p} \leq \alpha \leq(\gamma+1) p .
$$

Proof. Since $\mathcal{E}_{\alpha}^{q}(\Omega) \subset \mathcal{E}_{\alpha}^{r}(\Omega)$ for all $1 \leq r \leq q$ we may assume $f \in \mathcal{E}_{\alpha}^{\tilde{q}}(\Omega)$. Using Hölder inequality, Lemma 1.1 (with $r=p$ ), and noting that

$$
\tilde{q}^{\prime}=\frac{\tilde{q}}{\tilde{q}-1}=\frac{(N-\alpha) p}{N-(\gamma+1) p}=p_{\alpha, \gamma}^{*}
$$

we obtain

$$
\left|\int_{\Omega} f \phi d x\right| \leq\|f\|_{(\alpha, \tilde{q})}\left(\int_{\Omega}|\phi|^{\tilde{q}^{\prime}}|x|^{-\alpha} d x\right)^{1 / \tilde{q}^{\prime}} \leq\|f\|_{(\alpha, \tilde{q})}\|\phi\|_{\gamma, p},
$$

for all $\varphi \in \mathcal{C}_{0}^{\infty}(\Omega)$. We conclude the proof by a standard density argument.

As we said in the introduction, a measurable function $u$ such that $T_{t}(u) \in$ $\mathcal{D}_{0, \gamma}^{1, p}(\Omega)$, for all $t>0$, does not necessarily belong to $W_{0}^{1,1}(\Omega)$, nor to $L^{1}(\Omega)$. However, it is possible to define its weak gradient as the unique function $v$ satisfying condition (10) below. The weak gradient of $u$ is still denoted by $\nabla u$. The following result, proved in [1], introduces this notion.

Lemma 2.4. If $u$ is a measurable function such that $T_{t}(u) \in \mathcal{D}_{0, \gamma}^{1, p}(\Omega)$, for all $t>0$, then there exists a unique measurable function $v: \Omega \longrightarrow \mathbb{R}^{N}$ such that

$$
\nabla T_{t} u=v \chi_{\{|u|<t\}} \quad \text { for a.e. } x \in \Omega \text { and for all } t>0,
$$

where $\chi_{E}$ denotes the characteristic function of a measurable set $E$.

Finally, taking an adequate test function in the entropy condition (5) we obtain the following useful inequality.

Lemma 2.5. Assume that $u$ is the entropy solution of (1) then

$$
\frac{1}{t} \int_{\{s<|u| \leq s+t\}}|\nabla u|^{p}|x|^{-\gamma p} d x \leq \int_{\{|u|>s\}}|f(x)| d x .
$$

for all $s, t>0$. 
ProOF. The result follows taking $v=T_{s}(u)$ in the entropy condition (5).

\section{Estimates in Lebesgue spaces}

In order to prove Theorem 1.3, first we obtain some estimates for the distribution function of the entropy solution to problem (1). We obtain such estimates using Lemmas 2.2 and 2.5.

Lemma 3.1. Let $\alpha$ be any real number such that

$$
\frac{N \gamma p}{N-p} \leq \alpha \leq(\gamma+1) p
$$

Assume $\gamma<(N-p) / p$ and $f \in \mathcal{E}_{\alpha}^{q}(\Omega)$ for some $q \geq 1$. Consider $u$, then entropy solution of (1), let $r_{1}$ be defined in (6), and

$$
V_{\alpha}(t):=\int_{\{|u|>t\}}|x|^{-\alpha} d x .
$$

There exists a positive constant $C$ depending only on $N, \alpha, \gamma$, and $p$ such that the following assertions hold:

(i) If $\alpha<(\gamma+1) p$ and $q>(N-\alpha) /(\gamma p+p-\alpha)$ then $V_{\alpha}(t)=0$ for a.e.

$$
t \geq t^{*}:=-r_{1} C^{p^{\prime}}\|f\|_{(\alpha, q)}^{\frac{1}{p-1}}|\Omega|_{\alpha}^{-\frac{1}{r_{1}}}
$$

(ii) If $\alpha<(\gamma+1) p$ and $q=(N-\alpha) /(\gamma p+p-\alpha)$ then

$$
V_{\alpha}(t) \leq|\Omega|_{\alpha} \exp \left(-\frac{t}{C^{p^{\prime}}\|f\|_{(\alpha, q)}^{\frac{1}{p-1}}}\right) \quad \text { for a.e. } t>0 \text {. }
$$

(iii) If either, $\alpha=(\gamma+1) p$, or $\alpha<(\gamma+1) p$ and $1 \leq q<(N-\alpha) /(\gamma p+p-\alpha)$, then

$$
V_{\alpha}(t) \leq\left(A+\frac{t}{B}\right)^{-r_{1}} \quad \text { for a.e. } t>0,
$$

where

$$
A:=|\Omega|_{\alpha}^{-\frac{1}{r_{1}}} \quad \text { and } \quad B:=r_{1} C^{p^{\prime}}\|f\|_{(\alpha, q)}^{\frac{1}{p-1}}
$$


Proof. Let $E_{s, t}:=\{s<|u| \leq s+t\}$ for $s, t>0$, and

$$
\left|E_{s, t}\right|_{\alpha}:=\int_{\{s<|u| \leq s+t\}}|x|^{-\alpha} d x .
$$

From Lemma 2.5 and Hölder inequality, we obtain

$$
\int_{E_{s, t}}|\nabla u|^{p}|x|^{-\gamma p} d x \leq t\|f\|_{(\alpha, q)} V_{\alpha}(s)^{1 / q^{\prime}} \quad \text { for all } s, t>0 .
$$

On the other hand, using Jensen inequality, we have

$$
\frac{1}{\left|E_{s, t}\right|_{\alpha}^{p-1}}\left(\int_{E_{s, t}}|\nabla u||x|^{-\left(\gamma-\frac{\alpha}{p}+\alpha\right)} d x\right)^{p} \leq \int_{E_{s, t}}\left(|\nabla u||x|^{-\left(\gamma-\frac{\alpha}{p}\right)}\right)^{p}|x|^{-\alpha} d x
$$

for all $s, t>0$. From the last inequality and (14), we obtain

$$
\left(\frac{1}{t} \int_{E_{s, t}}|\nabla u \| x|^{-\left(\gamma-\frac{\alpha}{p}+\alpha\right)} d x\right)^{p} \leq\|f\|_{(\alpha, q)} V_{\alpha}(s)^{1 / q^{\prime}}\left(\frac{\left|E_{s, t}\right|_{\alpha}}{t}\right)^{p-1},
$$

for all $s, t>0$.

Let $\psi_{s, t}=T_{t}\left(u-T_{s} u\right)$ and note that $\nabla \psi_{s, t}=(\nabla u) \chi_{E_{s, t}}$. By Lemma 2.2, we have

$$
\begin{aligned}
\left(\int_{\Omega}\left|\psi_{s, t}\right|^{r}|x|^{-\alpha} d x\right)^{\frac{1}{r}} & \leq C \int_{\Omega}\left|\nabla \psi_{s, t}\right||x|^{-\left(\gamma-\frac{\alpha}{p}+\alpha\right)} d x \\
& =C \int_{E_{s, t}}|\nabla u||x|^{-\left(\gamma-\frac{\alpha}{p}+\alpha\right)} d x
\end{aligned}
$$

for all $s, t>0$, where

$$
r=\frac{N-\alpha}{N-\frac{\gamma p-\alpha}{p}-\alpha-1}, \quad \frac{N \gamma p}{N-p} \leq \alpha \leq(\gamma+1) p,
$$

and $C$ is a constant depending only on $N, \alpha, \gamma$, and $p$. Therefore, using (15), we obtain

$$
\left(\int_{\{|u|>s\}}\left(\frac{\left|\psi_{s, t}\right|}{t}\right)^{r}|x|^{-\alpha} d x\right)^{\frac{p}{r}} \leq C^{p}\|f\|_{(\alpha, q)} V_{\alpha}(s)^{1 / q^{\prime}}\left(\frac{\left|E_{s, t}\right|_{\alpha}}{t}\right)^{p-1},
$$

for all $s, t>0$. Letting $t \rightarrow 0$ we conclude that

$$
V_{\alpha}(s)^{p / r} \leq C^{p}\|f\|_{(\alpha, q)} V_{\alpha}(s)^{1 / q^{\prime}}\left(-V_{\alpha}^{\prime}(s)\right)^{p-1}, \quad \text { for a.e. } s>0,
$$


and then

$$
1 \leq C^{p^{\prime}}\|f\|_{(\alpha, q)}^{\frac{1}{p-1}} V_{\alpha}(s)^{-1-\frac{1}{r_{1}}}\left(-V_{\alpha}^{\prime}(s)\right), \quad \text { for a.e. } s>0
$$

where

$$
\frac{1}{r_{1}}=\frac{N-\alpha-(\gamma p+p-\alpha) q}{(p-1)(N-\alpha) q} .
$$

Recall the assumption $\alpha \leq(\gamma+1) p<N$. We study the following different cases:

1.- If $\alpha<(\gamma+1) p$ and $q>(N-\alpha) /(\gamma p+p-\alpha)$, i.e., $r_{1}<0$, then integrating (16) in $(0, t)$ we get

$$
t \leq-r_{1} C^{p^{\prime}}\|f\|_{(\alpha, q)}^{\frac{1}{p-1}}\left(|\Omega|_{\alpha}^{-\frac{1}{r_{1}}}-V_{\alpha}(t)^{-\frac{1}{r_{1}}}\right) .
$$

We conclude that $V_{\alpha}(t)=0$ if $t \geq t^{*}$, where $t^{*}$ is defined in (13).

2.- If $\alpha<(\gamma+1) p$ and $q=(N-\alpha) /(\gamma p+p-\alpha)$, i.e., $1 / r_{1}=0$, then we obtain the assertion integrating (16) in $(0, t)$.

3.- If either, $\alpha=(\gamma+1) p$, or $\alpha<(\gamma+1) p$ and $q<(N-\alpha) /(\gamma p+p-\alpha)$, i.e., $r_{1}>0$, we conclude as in the previous cases.

Remark 3.2. The constant $C$ appearing in Lemma 3.1 is the constant $D$ in (8) replacing $\gamma$ by $\gamma+\alpha-\alpha / p$. This explains the relation between (9) and (11).

Now, we prove Theorem 1.3 as an easy consequence of Lemma 3.1.

Proof of Theorem 1.3. Part (i) follows directly from Lemma 3.1(i). Parts (ii) and (iii) follow from Lemma 3.1(ii)-(iii) by noting

$$
\int_{\Omega}|u|^{r}|x|^{-\alpha} d x=r \int_{0}^{\infty} s^{r-1} V_{\alpha}(s) d s .
$$

\section{Estimates for the gradient}

In this section we prove Theorem 1.4 using Lemma 2.5 and the estimates obtained in Lemma 3.1. 
Proof of Theorem 1.4. Let $u$ be the entropy solution of (1) and let $V_{\alpha}$ be defined in (12). From Lemma 2.5 and Hölder inequality, we have

$$
\frac{1}{t} \int_{\{s<|u| \leq s+t\}}|\nabla u|^{p}|x|^{-\gamma p} d x \leq \int_{\{|u|>s\}}|f(x)| d x \leq\|f\|_{(\alpha, q)} V_{\alpha}(s)^{1 / q^{\prime}}
$$

for all $s, t>0$. Letting $t \searrow 0$ we obtain

$$
\frac{d}{d s} \int_{\{|u| \leq s\}}|\nabla u|^{p}|x|^{-\gamma p} d x \leq\|f\|_{(\alpha, q)} V_{\alpha}(s)^{1 / q^{\prime}}, \quad \text { for a.e. } s>0
$$

and integrating the last expression in $(0, t)$ we get

$$
\int_{\{|u| \leq t\}}|\nabla u|^{p}|x|^{-\gamma p} d x \leq\|f\|_{(\alpha, q)} \int_{0}^{t} V_{\alpha}(s)^{1 / q^{\prime}} d s .
$$

(i) Assume $\max \{1, \bar{q}\}<q<\tilde{q}$. Let $r_{1}$ and $r:=r_{2}$ be defined in (6) and (7), respectively, and note that $1<r<p$. Let

$$
\delta:=\frac{(p-r) \alpha}{p r}, \quad \beta:=\gamma+\delta, \quad \text { and } \quad s:=\frac{(p-r) r_{\alpha, \gamma+\delta}^{*}}{r}
$$

where $r_{\alpha, \gamma+\delta}^{*}$ is the critical Hardy-Sobolev exponent defined in Lemma 1.1.

We note that $0<s<1$ and there exists a constant $C$ such that

$$
\sum_{k=0}^{n} \frac{1}{(1+k)^{s}} \leq C\left(1+n^{1-s}\right), \quad \text { for all } n \in \mathbb{N}
$$

Let $M \in \mathbb{N}$ and define $v:=T_{M+1}(u)$. By Hölder inequality, we obtain

$$
\int_{\Omega}|\nabla v|^{r}|x|^{-\beta r} d x \leq\left(\int_{\Omega} \frac{|\nabla v|^{p}}{(1+|v|)^{s}}|x|^{-p \gamma} d x\right)^{\frac{r}{p}}\left(\int_{\Omega}(1+|v|)^{\frac{r s}{p-r}}|x|^{-\alpha} d x\right)^{1-\frac{r}{p}} \quad \text { (20) }
$$


Now, using (17), (19), and Hölder inequality, we estimate the first integral on the right-hand side of (20) as follows

$$
\begin{aligned}
& \int_{\Omega} \frac{|\nabla v|^{p}}{(1+|v|)^{s}}|x|^{-p \gamma} d x \leq \sum_{k=0}^{M} \frac{1}{(1+k)^{s}} \int_{\{k \leq|u|<k+1\}}|\nabla v|^{p}|x|^{-p \gamma} d x \\
& \leq \sum_{k=0}^{M} \frac{1}{(1+k)^{s}} \sum_{n=k}^{+\infty} \int_{\{n \leq|u|<n+1\}}|f| d x \\
& =\sum_{n=0}^{M} \int_{\{n \leq|u|<n+1\}}|f| \sum_{k=0}^{n} \frac{1}{(1+k)^{s}} d x+\sum_{n=M+1}^{+\infty} \int_{\{n \leq|u|<n+1\}}|f| \sum_{k=0}^{M} \frac{1}{(1+k)^{s}} d x \\
& \leq \sum_{n=0}^{M} \int_{\{n \leq|u|<n+1\}}|f| C\left(1+|v|^{1-s}\right) d x+\sum_{n=M+1}^{+\infty} \int_{\{n \leq|u|<n+1\}}|f| C\left(1+|v|^{1-s}\right) d x \\
& \leq C\|f\|_{1}+C \int_{\Omega}|f||v|^{1-s} d x \\
& \leq C\|f\|_{(\alpha, q)}\left[|\Omega|_{\alpha}^{\frac{1}{q^{\prime}}}+\left(\int_{\Omega}|v|^{(1-s) q^{\prime}}|x|^{-\alpha} d x\right)^{\frac{1}{q^{\prime}}}\right] .
\end{aligned}
$$

Using this inequality in (20) and noting that $(1-s) q^{\prime}=r_{\alpha, \beta}^{*}=s r /(p-r)$, we obtain

$$
\begin{aligned}
& \int_{\Omega}|\nabla v|^{r}|x|^{-\beta r} d x \\
& \leq C\|f\|_{(\alpha, q)}^{\frac{r}{p}}\left(|\Omega|_{\alpha}^{\frac{1}{q^{\prime}}}+\left(\int_{\Omega}|v|^{(1-s) q^{\prime}}|x|^{-\alpha} d x\right)^{\frac{1}{q^{\prime}}}\right)^{\frac{r}{p}}\left(\int_{\Omega}(1+|v|)^{\frac{s r}{p-r}}|x|^{-\alpha} d x\right)^{1-\frac{r}{p}} \\
& \leq C\|f\|_{(\alpha, q)}^{\frac{r}{p}}\left(1+\int_{\Omega}|v|^{r_{\alpha, \beta}^{*}}|x|^{-\alpha} d x\right)^{1-\frac{r}{p q}} .
\end{aligned}
$$

Finally, since

$$
\frac{r}{r_{\alpha, \beta}^{*}}>1-\frac{r}{p q}
$$

we obtain, by Hardy-Sobolev inequality (3), that

$$
\int_{\Omega}|\nabla v|^{r}|x|^{-\beta r} d x=\int_{\{|u| \leq M+1\}}|\nabla u|^{r}|x|^{-\beta r} d x \leq C \quad \text { for all } M \geq 0,
$$

where $C>0$ is a constant independent of $u$ and $M$. In particular, $u \in \mathcal{D}_{0, \beta}^{1, r_{2}}(\Omega)$.

The last assertion of part (i) follows directly from (3). 
(ii) Let $\tau_{1}:=p q /\left(r_{1}+q\right)$ and $\tau_{2}:=(p \gamma-\alpha) \tau_{1} / p$. Assume $1 \leq q<\tilde{q}$ and note that $-r_{1} / q^{\prime}+1>0$. Since $\alpha \leq(\gamma+1) p<N$ and

$$
q<\tilde{q}=\frac{N-\alpha}{(p-1)(N-\alpha)+(\gamma+1) p-\alpha},
$$

we are under the assumptions of Lemma 3.1(iii). Therefore, using (18) and the lemma, we estimate

$$
\begin{aligned}
& \int_{\left\{|x|^{-\tau_{2}}|\nabla u|^{\left.\tau_{1}>t\right\}}\right.} t^{r_{1}}|x|^{-\alpha} d x \\
& \leq \int_{\left\{|x|^{-\tau_{2}|\nabla u|^{\left.\tau_{1}>t\right\}}\{\{|u| \leq t\}}\right.} t^{r_{1}}\left(\frac{|x|^{-\tau_{2}}|\nabla u|^{\tau_{1}}}{t}\right)^{p / \tau_{1}}|x|^{-\alpha} d x+\int_{\{|u|>t\}} t^{r_{1}}|x|^{-\alpha} d x \\
& \leq\|f\|_{(\alpha, q)} t^{r_{1}-p / \tau_{1}} \int_{0}^{t} V_{\alpha}(s)^{1 / q^{\prime}} d s+t^{r_{1}} V_{\alpha}(t) \\
& \leq\|f\|_{(\alpha, q)} t^{r_{1} / q^{\prime}-1} \frac{B}{1-r_{1} / q^{\prime}}\left(A+\frac{t}{B}\right)^{1-\frac{r_{1}}{q^{\prime}}}+t^{r_{1}} V_{\alpha}(t) \\
& \leq C .
\end{aligned}
$$

In particular, $|x|^{-\tau_{2} r}|\nabla u|^{\tau_{1} r} \in L^{1}\left(\Omega,|x|^{-\alpha} d x\right)$ for all $0<r<r_{1}$, or equivalently,

$$
\int_{\Omega}|\nabla u|^{r}|x|^{-\left(\gamma+\frac{\alpha}{r}-\frac{\alpha}{p}\right) r} d x \leq C \quad \text { for all } 0<r<r_{2} .
$$

\section{Examples and applications}

In this section we introduce some examples showing the optimality of our results and the relation in some sense between problem (1) and the Hardy-Sobolev inequality (3).

5.1. Examples. 1.- We start with an example that shows the optimality of our results. Recall that $\alpha, \gamma$, and $N$ satisfy

$$
\frac{N \gamma p}{N-p} \leq \alpha \leq(\gamma+1) p<N
$$

Let $\theta$ be a real number such that $N-\theta-p \gamma>0$ and note that $f(x)=|x|^{-(\gamma p+\theta)} \in$ $L^{1}\left(B_{1}(0)\right)$. Let $u$ be the unique entropy solution to problem

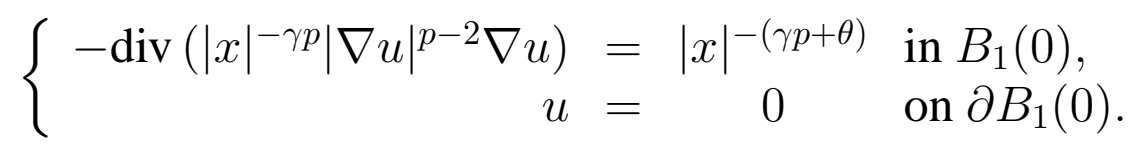


A direct computation shows that:

(1) If $\theta<p$, then

$$
u(x)=\frac{p-1}{(p-\theta)(N-\gamma p-\theta)^{\frac{1}{p-1}}}\left(1-|x|^{\frac{p-\theta}{p-1}}\right) .
$$

Hence, the solution is bounded.

(2) If $\theta=p$, then

$$
u(x)=\frac{1}{(N-p(\gamma+1))^{\frac{1}{p-1}}} \log \left(\frac{1}{|x|}\right) .
$$

In this case, one has that $u \in L^{r}\left(\Omega,|x|^{-\alpha} d x\right)$ for all $1 \leq r<+\infty$.

(3) If $\theta>p$, then

$$
u(x)=\frac{p-1}{(\theta-p)(N-\gamma p-\theta)^{\frac{1}{p-1}}}\left(|x|^{-\frac{\theta-p}{p-1}}-1\right) .
$$

In this last case, the solution $u$ is unbounded and

$$
u \in L^{r}\left(\Omega,|x|^{-\alpha} d x\right) \quad \text { if and only if } \quad 1 \leq r<(p-1) \frac{N-\alpha}{\theta-p} .
$$

By analyzing the above example we get easily that the regularity of $u$ obtained in Theorems 1.3 and 1.4 is sharp.

2.- Let us consider the following problem

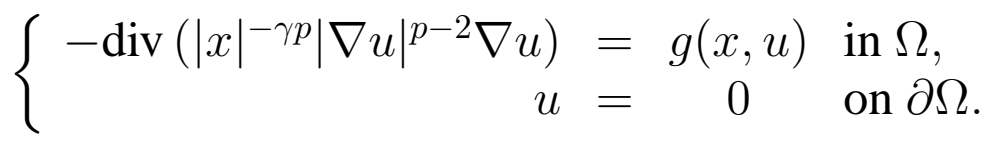

Assume that there exist positive constants $c$ and $m$ such that

$$
|g(x, t)| \leq c|x|^{-\alpha}(1+t)^{m-1} \quad \text { for all } t \geq 0 \text { and a.e. } x \in \Omega .
$$

In the particular case $\alpha=\gamma=0$, it is well known that every weak energy solution $u \in W_{0}^{1, p}(\Omega)$ of (22) is bounded whenever $m \leq p^{*}=N p /(N-p)$. In the general case one expect to obtain an analogous result, i.e., if $u \in \mathcal{D}_{0, \gamma}^{1, p}(\Omega)$ is a weak energy solution of (22) then $u \in L^{\infty}(\Omega)$ whenever $m \leq p_{\alpha, \gamma}^{*}$, where $p_{\alpha, \gamma}^{*}$ is the critical Hardy-Sobolev exponent appearing in Lemma 1.1. However, this problem remains open to our knowledge. In section 5.2 we will relate problems (22) and (1) in a particular case. 
The following explicit example shows that the exponent $p_{\alpha, \gamma}^{*}$ is optimal, in the sense that if $m>p_{\alpha, \gamma}^{*}$ then a solution $u \in \mathcal{D}_{0, \gamma}^{1, p}(\Omega)$ of (22) is not necessarily bounded. Set $g(x, t)=\lambda|x|^{-\alpha}(1+t)^{m-1}$, where

$$
m>1+(p-1) \frac{N-\alpha}{N-(\gamma+1) p}
$$

and

$$
\lambda:=\left(\frac{(\gamma+1) p-\alpha}{m-p}\right)^{p-1}\left[N-\frac{(\gamma p+p-\alpha)(m-1)+\alpha(m-p)}{m-p}\right] .
$$

Note that $m>p$ and $\lambda>0$ since (23). An easy computation shows that

$$
U(x)=|x|^{-\tau}-1, \quad \text { where } \quad \tau=\frac{(\gamma+1) p-\alpha}{m-p},
$$

is an entropy solution of (22). Moreover, $U \in \mathcal{D}_{0, \gamma}^{1, p}(\Omega)$ if and only if $m>p_{\alpha, \gamma}^{*}$. Therefore, if $m>p_{\alpha, \gamma}^{*}$ then $U \in \mathcal{D}_{0, \gamma}^{1, p}(\Omega)$ is an unbounded weak energy solution of (22). See [7] for more details in the case $\alpha=\gamma=0$.

5.2. Applications. We consider now the case where $p=2$ with go back to the result obtained in [6]. Let $u$ be the entropy solution to problem

$$
\left\{\begin{aligned}
-\operatorname{div}\left(|x|^{-2 \gamma} \nabla u\right) & =a \frac{u}{|x|^{2(\gamma+1)}}+g & & \text { in } \Omega, \\
u & = & 0 &
\end{aligned}\right.
$$

where $a<\Lambda_{N, \gamma}:=(N-2(\gamma+1))^{2} / 4$. We set $w(x)=|x|^{\beta} u(x)$, where

$$
\beta:=\frac{N-2(\gamma+1)}{2}-\sqrt{\Lambda_{N, \gamma}-a}>0,
$$

then it is clear that $w$ solves problem

$$
\left\{\begin{aligned}
-\operatorname{div}\left(|x|^{-2(\gamma+\beta)} \nabla w\right) & =|x|^{-\beta} g & & \text { in } \Omega \\
w & =0 & & \text { on } \partial \Omega .
\end{aligned}\right.
$$

As an immediate consequence of Theorems 1.3 and 1.4 we get the next corollary.

Corollary 5.1. Assume that $|x|^{-\beta} g \in \mathcal{E}_{\alpha}^{q}(\Omega)$ for some $\alpha$ and $q$ satisfying (2). Let

$$
r_{1}:=\frac{(N-\alpha) q}{N-\alpha-(2(\gamma+\beta)+2-\alpha) q} .
$$


Let $u$ be the entropy solution of (24), then there exists a positive constant $C$ depending only on $\Omega, N, \alpha, \gamma$ and $\beta$ such that the following assertions hold:

(i) If $\alpha<2(\gamma+\beta+1)$ and $q>(N-\alpha) /(2(\gamma+\beta)+2-\alpha)$, then $u|x|^{\beta}=$ $w \in L^{\infty}(\Omega)$. Moreover,

$$
\left\|u|x|^{\beta}\right\|_{\infty} \leq C\left\||x|^{-\beta} g\right\|_{(\alpha, q)} .
$$

(ii) If $\alpha<2(\gamma+\beta+1)$ and $q=(N-\alpha) /(2(\gamma+\beta)+2-\alpha)$, then $u|x|^{\beta} \in$ $L^{r}\left(\Omega,|x|^{-\alpha} d x\right)$, for all $1 \leq r<+\infty$. Moreover,

$$
\left(\int_{\Omega} u^{r}|x|^{r \beta-\alpha} d x\right)^{\frac{1}{r}} \leq C\left\||x|^{-\beta} g\right\|_{(\alpha, q)} .
$$

(iii) If either, $\alpha=2(\gamma+\beta+1)$, or $\alpha<2(\gamma+\beta+1)$ and $1 \leq q<(N-\alpha) /(2(\gamma+$ $\beta)+2-\alpha)$, then $u^{r}|x|^{\beta r} \in L^{1}\left(\Omega,|x|^{-\alpha} d x\right)$, for all $0<r<r_{1}$. Moreover,

$$
\left(\int_{\Omega}|u|^{r}|x|^{r \beta-\alpha} d x\right)^{\frac{1}{r}} \leq C\left\|g|x|^{-\beta}\right\|_{(\alpha, q)}, \quad \text { for all } 0<r<r_{1},
$$

where in this case the constant $C$ depends also on $q$.

Moreover, by Theorem 1.4 one gets the corresponding integrability of $\left|\nabla\left(|x|^{\beta} u\right)\right|$.

\section{References}

[1] B. Abdellaoui, I. Peral, On quasilinear elliptic equations related to some Caffarelli-Kohn-Nirenberg inequalities, Comm. Pure Appl. Anal. 2 (2003), 539-566.

[2] A. Alvino, L. Boccardo, V. Ferone, L. Orsina, G. Trombetti, Existence results for nonlinear elliptic equations with degenerate coercivity, Ann. Mat. Pura Appl. (4) 182 (2003), 53-79.

[3] P. Bénilan, L. Boccardo,T. Gallouët, R. Gariepy, M. Pierre, J. Vázquez, An $L^{1}$-theory of existence and uniqueness of solutions of nonlinear elliptic equations, Ann. Scuola Norm. Sup. Pisa Cl. Sci. (4) 22 (1995), 241-273.

[4] L. Boccardo, T. Gallouët, Nonlinear elliptic equations with right-hand side measures, Comm. Partial Differential Equations 14 (1989), 663-680.

[5] L. Boccardo, T. Gallouët, Nonlinear elliptic equations with right-hand side measures, Comm. Partial Differential Equations 17 (1992), 641-655.

[6] L. Boccardo, L. Orsina, I. Peral, A remark on existence and optimal summability of elliptic problems involving Hardy potential, to appear in Dynam. Systems Appl.

[7] X. Cabré, M. Sanchón, Semi-stable and extremal solutions of reaction equations involving the $p$ Laplacian, to appear in Commun. Pure Appl. Anal.

[8] L. Caffarelli, R. Kohn, L. Nirenberg, First order interpolation inequalities with weights, Compos. Math. 53 (1984), 259-275.

[9] E. Colorado, I. Peral, Eigenvalues and bifurcation for elliptic equations with mixed Dirichlet-Neumann boundary conditions related to Caffarelli-Kohn-Nirenberg inequalities. Topol. Methods Nonlinear Anal. 23 (2004), 239-273.

[10] E. De Giorgi, Sulla differenziabillitá e l'analiticitá delle estremali degli integrali multipli regulari, Mem. Accad. Sci. Torino Cl. Sci. Fis. Mat. Natur. (3) (1957), 25-43. 
[11] E. DiBenedetto, J. Manfredi, On the higher integrability of the gradient of weak solutions of certain degenerate elliptic systems, Amer. J. Math. 115 (1993), 1107-1134.

[12] V. Felli, M. Schneider, A note on regularity of solutions to degenerate elliptic equations of CaffarelliKohn-Nirenberg type, Adv. Nonlinear Stud. 3 (2003), 431-443.

[13] N. Grenon, $L^{r}$ estimates for degenerate elliptic problems, Potential Anal. 16 (2002), 387-392.

[14] J. Kinnunen, S. Zhou, A boundary estimate for nonlinear equations with discontinuous coefficients, Differential Integral Equations 14 (2001), 475-492.

[15] J. Leray, J.L. Lions, Quelques résultats de Višik sur les problèmes elliptiques nonlinéaires par les méthodes de Minty-Browder, Bull. Soc. Math. France 93 (1965), 97-107.

[16] J. Moser, On Harnack's theorem for elliptic differential equations, Comm. Pure Appl. Math. 13 (1961), $577-591$.

[17] J. Moser, On Harnack's inequality for parabolic differential equations, Comm. Pure Appl. Math. 17 (1964), 101-134.

[18] J. Nash, Continuity of solution of parabolic and elliptic equations, Amer. J. Math. 80 (1958), 931-954.

[19] G. Talenti, Nonlinear elliptic equations, rearrangements of functions and Orlicz spaces, Ann. Mat. Pura Appl. (4) 120 (1979), 160-184.

BOUMEDIENE ABDELlAOUI

Universidad Autónoma de Madrid, Departamento de Matemáticas, 28049 - Madrid, Spain

E-mail address: boumediene.abdellaoui@uam.es

EDUARDO COLORADO

Dpto. de Análisis Matemático, Universidad de Granada, Campus Fuentenueva s/N, 18071 GRANADA, SPAIN

E-mail address: colorado@ugr.es

MANEL SANCHÓN

Centro de Matemática, Universidade de Coimbra, 3001-454 Coimbra,Portugal

E-mail address: msanchon@mat.uc.pt 